\title{
APPARENT HORIZONS IN VACUUM ROBINSON-TRAUTMAN SPACETIMES
}

\author{
E. W. M. CHOW ${ }^{1}$ and A. W.-C. LUN ${ }^{1}$
}

(Received 24 March 1995)

\begin{abstract}
Vacuum asymptotically flat Robinson-Trautman spacetimes are a well known class of spacetimes exhibiting outgoing gravitational radiation. In this paper we describe a method of locating the past apparent horizon in these spacetimes and discuss the properties of the horizon. We show that the past apparent horizon is non-timelike and that its surface area is a decreasing function of the retarded time. A numerical simulation of the apparent horizon is also discussed.
\end{abstract}

\section{Introduction}

Vacuum asymptotically flat Robinson-Trautman spacetimes have been the subject of much study since their discovery over thirty years ago [18]. They possess some very nice features which make them amenable to analysis. They are in some sense the simplest asymptotically flat solutions which exhibit gravitational radiation, albeit of a fairly specialised nature. The feature of the spacetime which simplifies analysis is that the full spacetime can be built up from the solution of a fourth order parabolic equation on a $2+1$ dimensional manifold. This is a consequence of the fact that the coordinate system uses a retarded time coordinate, so that in effect initial data is prescribed on a null hypersurface.

Early studies of the Robinson-Trautman equation related to the behaviour of solutions of the linearised equation [5,25]. Lukács et al. [10] studied the behaviour of solutions of the full nonlinear equation, using the concept of Lyapunov stability to establish that global solutions, if they existed, would converge to the static Schwarzschild equilibrium. A number of authors subsequently focused on the existence of such solutions. Schmidt [19] showed local existence of solutions for sufficiently differentiable

\footnotetext{
'Department of Mathematics, Monash University, Clayton 3168, Australia.

(C) Australian Mathematical Society 1999, Serial-fee code 0334-2700/99
} 
but otherwise arbitrary initial data. Rendall [17] showed global existence for sufficiently small initial data, antipodally symmetric on the sphere, and his proof was extended by Singleton [21] to remove the requirement of antipodal symmetry. Finally Chruściel [2] proved semi-global existence of solutions for arbitrary smooth initial data. Numerical studies by Perjés [15] and Singleton [20,22] demonstrate vividly the evolution of an initially perturbed spacetime to the steady state. It is interesting to note that the evolution equations exhibit exponential divergence from arbitrary initial data in the "backwards" time direction, and that no solutions exist in this direction [2]. It should be emphasised that these results, and the subsequent discussion, only apply to asymptotically flat Robinson-Trautman spacetimes with regular topological $S^{2}$ surfaces: Perjés and Hoenselaers [9] have shown that there exists a class of RobinsonTrautman spacetimes with cusp singularities which evolve to static $C$-metrics.

Thus the evolution of the Robinson-Trautman spacetimes would seem to be thoroughly understood. We would expect then that the behaviour of physical features of the spacetimes should not be too difficult to pin down. Near $\mathcal{J}^{+}$, the spacetimes, because of their algebraically degenerate structure, could reasonably be interpreted as describing purely outgoing radiation around a black hole source: for example the decaying tail of the radiation after the black hole has formed (note that there is no ingoing radiation, which means there can be no backscattering or similar self-interaction of the radiation field). However, in this paper, we will be studying the structure of past apparent horizons in vacuum Robinson-Trautman spacetimes; thus we will focus our attention on the behaviour of the "white hole" region of the spacetimes. Since these spacetimes evolve to the Schwarzschild geometry, the future apparent horizons coincide with the future event horizons at $u=\infty(r=2 m$, in Schwarzschild coordinates).

Our study of the apparent horizon structure of these spacetimes was initially motivated by an investigation of the stability of the related class of electrovac spacetimes, the Robinson-Trautman Einstein-Maxwell spacetimes, discussed elsewhere [11]. The asymptotically flat vacuum Robinson-Trautman spacetimes turn out to have a particularly well behaved apparent horizon which illustrates several general theorems concerning apparent horizons. There are in fact very few well understood exact solutions which can be used to illustrate such theorems - there being few examples of non-static black hole type solutions. The asymptotically flat Robinson-Trautman spacetimes, in some sense the simplest asymptotically flat spacetimes admitting gravitational radiation, should be a useful example for the study of radiating black hole spacetimes in general and many of the general theorems relating to non-stationary spacetimes. The basic equations and features of the spacetimes are outlined in Section 2. Section 3 contains the results concerning the past apparent horizon and its properties. Some numerical demonstration of these results is presented in Section 4. 


\section{Robinson-Trautman vacuum spacetimes}

2.1. Basic equations and notation The line element for the Robinson-Trautman class of spacetimes is given by:

$$
d s^{2}=2 H d u^{2}+2 d u d r-\frac{2 r^{2} d \zeta d \bar{\zeta}}{P^{2}}
$$

where $H=-r(\ln P)_{{ }_{u}}+\frac{1}{2} K-\frac{m}{r}$ and $K=\Delta(\ln P)$. The vacuum Robinson-Trautman spacetimes are foliated by a two parameter family of 2-surfaces $\mathcal{S}_{u, r}$ which, for the asymptotically flat case, have spherical topology. The operator $\Delta=2 P^{2} \partial_{\zeta \bar{\zeta}}$ is the Laplacian on these 2-surfaces. For the vacuum spacetimes, $m=m(u)$ and we can use a coordinate transformation on $u$ to make $m$ constant. We are interested in the $m>0$ case, as this gives rise to a spacetime with positive Bondi mass. The spacetime is then determined by the evolution of $P=P(u, \zeta, \bar{\zeta})$ on a background two-sphere. The evolution equation is often written as

$$
(\ln P)_{{ }_{u}}=-\frac{1}{12 m} \Delta K
$$

This equation, the Robinson-Trautman equation, is also known in the literature as the two-dimensional Calabi equation [24].

The steady state, corresponding to the Schwarzschild solution, is given by $P=$ $P_{0}=\frac{1}{\sqrt{2}}(a+b \zeta+\bar{b} \bar{\zeta}+c \zeta \bar{\zeta})$, where $a c-b \bar{b}=1$. Note that the equilibrium value of $P$ is not unique but includes a freedom corresponding to conformal motions on the sphere. This freedom was a contributing factor to the difficulty of proving the existence of solutions of the Robinson-Trautman equation [2]. The condition $a c-b \bar{b}=1$ normalises the Gaussian curvature of $S^{2}$ to $K=1$.

The function $P$ determines the induced 2-metric on each $\mathcal{S}_{u, r}$, which is given by

$$
g_{\mathcal{S}}=\frac{2 r^{2} d \zeta d \bar{\zeta}}{P^{2}}=e^{2 \Lambda} g_{0}
$$

where $g_{0}$ is the metric of $S^{2}$ and $e^{-\Lambda}=P / P_{0}$. It is possible to "factor out" the background $S^{2}$ geometry and write the Robinson-Trautman equation as

$$
e^{2 \Lambda} \Lambda_{{ }_{u}}=\frac{1}{12 m} \Delta_{0} K
$$

where $\Delta_{0}$ is the Laplacian on $S^{2}$. We thus solve the evolution equation on a background sphere, say at $r=1$. 
2.2. Conserved quantities and Lyapunov functionals Several conserved integrals are implied by the evolution equations. First, we have the conservation of surface area of $\mathcal{S}_{u, r}$ and the conservation of the "irreducible mass". As a consequence of this

$$
\begin{aligned}
& A_{\mathcal{S}}=\int_{\mathcal{S}_{\infty}} \omega_{1}=\int_{S^{2}} e^{2 \Lambda} \omega_{0}=4 \pi, \\
& M_{I}=\frac{1}{4 \pi} \int_{\mathcal{S}_{\infty}} m \omega_{1}=\frac{m}{4 \pi} \int_{S^{2}} e^{2 \Lambda} \omega_{0}=m .
\end{aligned}
$$

The Gaussian curvature, in terms of $\Lambda$, takes the form $K=e^{-2 \Lambda}\left(1-\Delta_{0} \Lambda\right)$ which, making use of Stokes' theorem, immediately gives the conservation of the Euler number for $\mathcal{S}_{u, r}$ :

$$
\chi_{S}=\frac{1}{2 \pi} \int_{S^{2}} K e^{2 \Lambda} \omega_{0}=2 .
$$

Singleton $[20,22]$ gave an expression for the Bondi-Sachs mass of the spacetime

$$
M_{B}=\frac{m}{4 \pi} \int_{S^{2}} e^{3 \Lambda} \omega_{0}
$$

which is manifestly positive and in fact is bounded below by the irreducible mass, since by the Hölder inequality

$$
\left(\int_{S^{2}} \omega_{0}\right)^{1 / 3}\left(\int_{S^{2}} e^{3 \Lambda} \omega_{0}\right)^{2 / 3} \geq \int_{S^{2}} e^{2 \Lambda} \omega_{0} .
$$

That the Bondi mass is monotonically decreasing can be shown by differentiating (4) and using the Robinson-Trautman equation:

$$
\frac{d}{d u} M_{B}=-\frac{1}{4 \pi} \int_{S^{2}} e^{\Lambda}\left(P_{0}^{2}\left(e^{-\Lambda}\right)_{\cdot \bar{\zeta}}\right)_{\cdot \bar{\zeta}}\left(P_{0}^{2}\left(e^{-\Lambda}\right)_{\zeta}\right)_{\zeta \zeta} \omega_{0} \leq 0 .
$$

These characteristics enabled Singleton $[20,22]$ to show that the Bondi Mass is a Lyapunov functional for the Robinson-Trautman evolution, complementing the earlier work of Lukács et al. [10] who showed that the integral $\int_{S^{2}} K^{2} e^{2 \Lambda} \omega_{0}$ is a Lyapunov functional for the Robinson-Trautman evolution. These Lyapunov functionals played a key role in Chruściel's semi-global existence proof [2].

\section{The past apparent horizon}

3.1. General remarks Apparent horizons in black hole spacetimes have been the subject of much study in recent times. The motivation for the study of the future or 
past apparent horizons is that they provide a locally characterisable indication of the presence of an event horizon (future) or particle horizon (past). Indeed, in cases where the event horizon or particle horizon cannot be properly defined, the apparent horizon may be the only useful definition of the "surface" of the black hole or white hole [8]. This arguably is the case in the Robinson-Trautman spacetimes, where $\mathcal{J}^{-}$cannot be properly defined due to the instability of the Robinson-Trautman equation in the negative $u$ direction [2]. Apparent horizons have been widely used in numerically generated spacetimes as an indicator of the presence of a black hole $[4,12]$ in lieu of being able to detect the event horizon in a local way. ${ }^{2}$ It is believed that the presence of an apparent horizon indicates the presence of a nearby event horizon (where it exists), and it has been shown that for all stationary black hole spacetimes the apparent and event horizons coincide [7]. Recent work has shown that the apparent horizon of a black hole has "thermodynamic" properties: in particular it has been shown that a future apparent horizon can only increase in area, while a past apparent horizon can only decrease in area, obeying a law like that of the first law of thermodynamics $[3,8]$.

The Robinson-Trautman spacetimes, as they settle down to the Schwarzschild solution as $u \rightarrow \infty$, would be expected to have a well behaved future apparent horizon coinciding with the event horizon. Due to the presence of outgoing radiation, we would expect the past apparent horizon to be more complex. As a general (and non rigorous) consideration, it can be seen quite clearly from the line element (1) that the curvature singularity at $r=0$ is a spacelike singularity, that is, that if the spacetime were complete in the past null direction (if $\mathcal{J}^{-}$were complete) then there would exist a particle horizon - the past counterpart of the event horizon (see Figure 1). Effectively, the past singularity being spacelike indicates that no null geodesics from $\mathcal{J}^{-}$can reach the singularity, if the spacetime is to be causally well-behaved. Because the particle horizon is a globally defined object, it does not exist in Robinson-Trautman spacetimes. However, the spacelike nature of the past singularity suggests that there should always be a past apparent horizon in these spacetimes, which we believe to be causally well-behaved.

\subsection{Apparent horizon equations The concept of an apparent horizon rests on} the definition of a marginally trapped surface. We define an outer past marginally trapped surface, $\mathcal{I}^{-}$, to be a spacelike 2-surface on which the ingoing future directed congruence of orthogonal null geodesics has vanishing divergence, while the outgoing future directed congruence of orthogonal null geodesics is diverging. We define the outer past apparent horizon, $\mathcal{H}^{-}$, to be a hypersurface $r=\Re(u, \zeta, \bar{\zeta})$ such that its intersection with each $u=u_{0}$ slice is an outer marginally past trapped 2-surface $\mathcal{I}_{u}^{-}$, that is, $\mathcal{H}^{-}$is foliated by the surfaces $\mathcal{I}_{u}^{-}$. The past apparent horizon is illustrated in

\footnotetext{
${ }^{2}$ In recent work, Anninos et al. [1] have been able to locate the event horizon in numerically generated spacetimes by integrating backwards from the stationary final state of the black hole.
} 


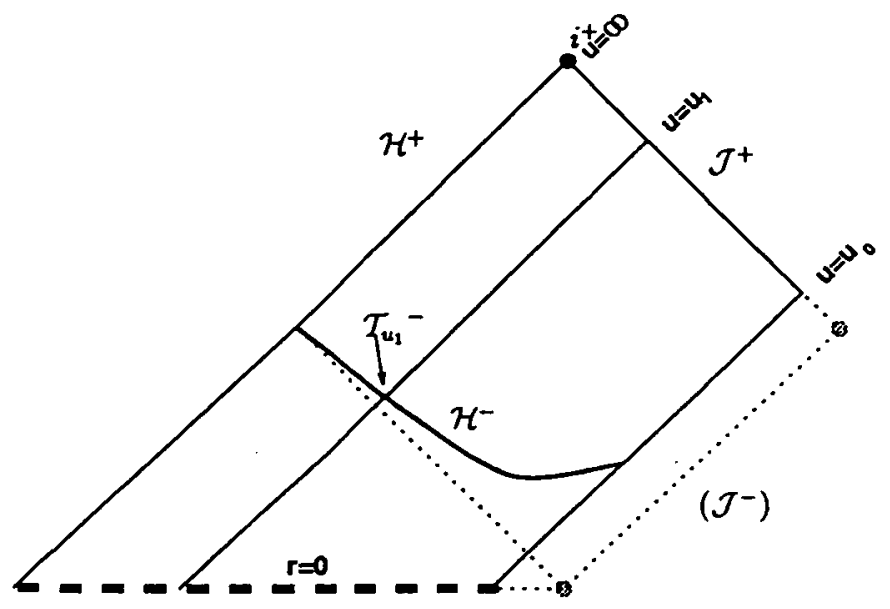

FIGURE 1. Penrose diagram for the Robinson-Trautman spacetime.

Figure 1.

The equations describing the apparent horizon are constructed as follows. Consider a null tetrad $\left(l^{a}, n^{a}, m^{a}, \bar{m}^{a}\right)$ for the spacetime with $l^{a}$ tangent to a congruence of outgoing shearfree diverging null geodesics, and $m^{a}$ and $\bar{m}^{a}$ lying on a family of 2-surfaces $\mathcal{I}_{u}^{-}$:

$$
\begin{aligned}
l^{a} & =\partial_{r}, \\
n^{a} & =\partial_{u}+\left[\frac{P^{2} \Re_{\zeta} \Re_{\bar{\zeta}}}{r^{2}}-H\right] \partial_{r}+\frac{P^{2} \Re_{\zeta}}{r^{2}} \partial_{\zeta}+\frac{P^{2} \Re_{\bar{\zeta}}}{r^{2}} \partial_{\bar{\zeta}}, \\
m^{a} & =\frac{P}{r} \partial_{\bar{\zeta}}+\frac{P \Re_{\bar{\zeta}}}{r} \partial_{r}, \\
\bar{m}^{a} & =\frac{P}{r} \partial_{\zeta}+\frac{P \Re_{\zeta}}{r} \partial_{r},
\end{aligned}
$$

where $H$ is as in (1) and $r=\mathfrak{R}(u, \zeta, \bar{\zeta})$ defines the surfaces $\mathcal{I}_{u}^{-}$. The nonvanishing Newman-Penrose quantities are then given by

$$
\begin{aligned}
& \rho=-\frac{1}{r}, \quad \tau=\bar{\pi}=\bar{\alpha}+\beta=-\frac{P \Re_{\bar{\zeta}}}{r^{2}}, \quad \beta=-\frac{P_{\cdot \bar{\zeta}}}{2 r}, \\
& \lambda=\left(\partial_{\zeta}+\Re_{r_{\zeta}} \partial_{r}\right)\left(\frac{P^{2} \Re_{\zeta_{\zeta}}}{r^{2}}\right) \text {, } \\
& \mu=-\frac{1}{2 r}\left[K-\frac{2}{r}\left(m+P^{2} \mathfrak{R}_{{ }_{\zeta \zeta}}\right)+\frac{1}{r^{2}}\left(2 P^{2} \Re_{{ }_{\zeta}} \Re{ }_{, \zeta \zeta}\right)\right],
\end{aligned}
$$

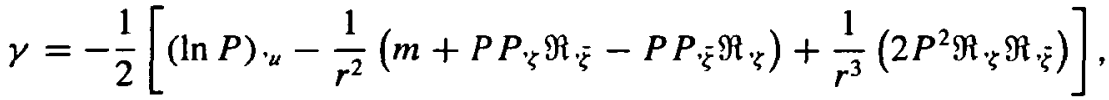




$$
\begin{aligned}
\nu & =\frac{P}{r}\left(\partial_{\zeta}+\Re_{\zeta \zeta} \partial_{r}\right)\left(\Re_{{ }_{u}}-r(\ln P)_{{ }_{u}}+\frac{K}{2}-\frac{m}{r}+\frac{P^{2} \Re_{{ }_{\zeta}} \Re_{{ }_{\zeta}}}{r^{2}}\right), \\
\Psi_{2} & =-\frac{m}{r^{3}}, \\
\Psi_{3} & =-\frac{P K_{{ }_{\zeta}}}{2 r^{2}}-\frac{3 P m \Re_{{ }_{\zeta}}}{r^{4}}, \\
\Psi_{4} & =-\frac{\left[P^{2}(\ln P)_{{ }_{u \zeta}}\right]_{\zeta \zeta}}{r}+\frac{\left(P^{2} K_{{ }_{\zeta}}\right)_{{ }_{\zeta}}}{2 r^{2}}-\frac{2 P^{2} K_{\cdot_{\zeta}} \Re_{{ }_{\zeta}}}{r^{3}}-\frac{6 P^{2} m\left(\Re_{r_{\zeta}}\right)^{2}}{r^{5}} .
\end{aligned}
$$

From (6) and (7) it can be seen that the null vector $l^{a}$ is future directed, outward pointing, geodetic $(\kappa=0)$, diverging $(\rho=-1 / r)$, null and orthogonal to the 2-surface $\mathcal{I}_{u}^{-}$. That is, $l^{a}$ is tangent to the outgoing congruence of null geodesics normal to $\mathcal{I}_{u}^{-}$. The tetrad vector $n^{a}$ will be tangent at $\mathcal{I}_{u}^{-}$to the ingoing congruence of null geodesics normal to $\mathcal{I}_{u}^{-}$. The divergence of $n^{a}$ is given by $\operatorname{Re}(\mu)(=\mu$ in this case). Thus the divergence of the ingoing congruence of null geodesics is given on $\mathcal{I}_{u}^{-}$by $\tilde{\mu}$, where the tilde is used here and subsequently to denote the restriction of an $r$-dependent quantity to the hypersurface $r=\mathfrak{R}(u, \zeta, \bar{\zeta})$. If $\tilde{\mu}=0, \mathcal{I}_{u}^{-}$are marginally past-trapped 2-surfaces $\mathcal{I}_{u}^{-}$and the hypersurface $r=\mathfrak{N}(u, \zeta, \bar{\zeta})$ is the past apparent horizon $\mathcal{H}^{-}$. This condition gives us the first horizon equation:

$$
K-\frac{2 m}{\Re}-\Delta(\ln \Re)=0 .
$$

This equation is attributed to Penrose [13]. Tod [24] examined it in more detail, proving that (8) has a unique $C^{\infty}$ solution, given a $C^{\infty}$ background $\mathcal{S}_{u, r}$, and also that the surface $\mathcal{I}_{u}^{-}$defined by the solution is in fact the outer boundary of past-trapped surfaces on $u=u_{0}$.

The other equations are found by examining the embedding of the past apparent horizon in the spacetime; in particular by considering the normal vector to the apparent horizon.

Let $N_{a}$ be a one-form on $\mathcal{H}^{-}$defined by $N_{a}=-\Re_{{ }_{u}} d u+d r-\Re_{{ }_{\zeta}} d \zeta-\Re_{{ }_{\zeta}} d \bar{\zeta}$. Hence $N^{a}:=g^{a b} N_{b}$ is a vector orthogonal to $\mathcal{H}^{-}$and is given by

$$
\begin{aligned}
N^{a} & =\tilde{n}^{a}-\left(\Re_{{ }_{u}}+\tilde{H}+\frac{P^{2} \mathfrak{R}_{{ }_{\zeta}} \mathfrak{R} \cdot \tilde{\zeta}}{\mathfrak{R}^{2}}\right) \tilde{l}^{a} \\
& =\partial_{u}-\left(\Re_{{ }_{u}}+2 \tilde{H}\right) \partial_{r}+\frac{P^{2} \mathfrak{R}_{{ }_{\zeta}}}{\mathfrak{R}^{2}} \partial_{\zeta}+\frac{P^{2} \mathfrak{R}_{\cdot_{\zeta}}}{\mathfrak{N}^{2}} \partial_{\bar{\zeta}} .
\end{aligned}
$$

Since the complex null vectors $\tilde{m}^{a}$ and $\tilde{\bar{m}}^{a}$ are tangent to the 2-surfaces $\mathcal{I}_{u}^{-}$which foliate the hypersurface $\mathcal{H}^{-}$, the null vectors $\tilde{l}^{a}$ and $\tilde{n}^{a}$ are orthogonal to $\mathcal{I}_{\dot{u}}^{-}$. It can be seen that the vector

$$
Z^{a}=\tilde{n}^{a}+\left(\Re_{\cdot_{u}}+\tilde{H}+\frac{P^{2} \mathfrak{R}_{\zeta} \mathfrak{N}_{\cdot \bar{\zeta}}}{\mathfrak{R}^{2}}\right) \tilde{l}^{a}
$$




$$
=\partial_{u}+\left(\Re_{{ }_{u}}+\frac{2 P^{2} \Re_{{ }_{\zeta}} \Re_{{ }_{\zeta}}}{\mathfrak{R}^{2}}\right) \partial_{r}+\frac{P^{2} \Re_{\bar{\zeta}}}{\Re^{2}} \partial_{\zeta}+\frac{P^{2} \Re{ }_{\cdot \zeta}}{\mathfrak{R}^{2}} \partial_{\bar{\zeta}}
$$

is orthogonal to $N^{a}$ and therefore is tangent to the hypersurface $\mathcal{H}^{-}$. The "magnitude" of $N^{a}$ and $Z^{a}$, from (9) and (10), is given by

$$
N_{a} N^{a}=-Z_{a} Z^{a}=-2\left(\Re_{{ }_{u}}+\tilde{H}+\frac{P^{2} \mathfrak{R}_{{ }_{\zeta}} \mathfrak{R} \cdot \bar{\xi}}{\Re^{2}}\right) .
$$

Since from (8) $\bar{\mu}=0$ on each $\mathcal{I}_{u}^{-}$, the directional derivative of $\tilde{\mu}$ along the vector $Z^{a}$ tangent to $\mathcal{H}^{-}$must vanish. Equations (10) and (11) then imply

$$
Z^{a} \nabla_{a} \tilde{\mu}=\tilde{n}^{a} \nabla_{a} \tilde{\mu}-\frac{1}{2}\left(N_{b} N^{b}\right) \tilde{l}^{a} \nabla_{a} \tilde{\mu}=0
$$

Substituting (7) into the Newman-Penrose equations [14] gives

$$
\begin{aligned}
\tilde{n}^{a} \nabla_{a} \tilde{\mu}= & \tilde{m}^{a} \nabla_{a} \tilde{v}+\tilde{v}(-\tilde{\tau}+\tilde{\bar{\alpha}}+3 \tilde{\beta})+\tilde{\pi} \tilde{\bar{\nu}}-\tilde{\lambda} \tilde{\bar{\lambda}}-2 \tilde{\phi}_{2} \tilde{\bar{\phi}}_{2} \\
= & -\partial_{\zeta}\left(\frac{P^{2} \mathfrak{R}{ }_{\zeta \zeta}}{\mathfrak{R}^{2}}\right) \partial_{\bar{\zeta}}\left(\frac{P^{2} \mathfrak{R} \cdot \bar{\xi}}{\mathfrak{R}^{2}}\right) \\
& -\frac{P^{2}}{2 \mathfrak{R}}\left[\partial_{\zeta \bar{\zeta}}\left(\frac{N_{a} N^{a}}{\mathfrak{R}}\right)-\left(N_{a} N^{a}\right) \partial_{\zeta \bar{\zeta}}\left(\frac{1}{\mathfrak{R}}\right)\right], \\
\tilde{l}^{a} \nabla_{a} \tilde{\mu}= & \tilde{m}^{a} \nabla_{a} \tilde{\pi}+\tilde{\pi}(\tilde{\bar{\pi}}-\tilde{\bar{\alpha}}+\tilde{\beta})+\tilde{\Psi}_{2} \\
= & \frac{P^{2}}{\mathfrak{R}} \partial_{\zeta \bar{\zeta}}\left(\frac{1}{\mathfrak{R}}\right)-\frac{m}{\mathfrak{R}^{3}} .
\end{aligned}
$$

In (13) we have used $\tilde{\nu}=-\frac{P}{2 \Re}\left(N_{a} N^{a}\right)_{\zeta}$ and $\tilde{\lambda}=\left(\frac{P^{2} \Re_{\zeta}}{\Re^{2}}\right)_{\zeta} \cdot$ Combine (12), (13) and (14) to give

$$
\frac{1}{\mathfrak{R}} \Delta\left(\frac{N_{a} N^{a}}{\mathfrak{M}}\right)+2 \tilde{\Psi}_{2}\left(N_{a} N^{a}\right)=-4 \tilde{\bar{\lambda}} \tilde{\bar{\lambda}}
$$

To summarise, then, the apparent horizon in asymptotically flat Robinson-Trautman spacetimes can be described by the following equations:

$$
\begin{gathered}
K-\frac{2 m}{\mathfrak{R}}=\Delta(\ln \mathfrak{R}), \\
\frac{1}{\mathfrak{R}} \Delta\left(\frac{N_{a} N^{a}}{\mathfrak{R}}\right)-2 \frac{m}{\mathfrak{R}^{3}}\left(N_{a} N^{a}\right)=-4 \tilde{\lambda} \tilde{\bar{\lambda}} \quad \text { and } \\
N_{a} N^{a}=-2 \mathfrak{P}\left(\ln \frac{\mathfrak{R}}{P}\right)_{\cdot_{u}}-K+\frac{2 m}{\mathfrak{R}}-\frac{2 P^{2} \mathfrak{R}_{\zeta} \mathfrak{R} \cdot \tilde{\zeta}}{\mathfrak{R}^{2}} .
\end{gathered}
$$


The $\operatorname{Re}(\tilde{\mu})=0$ equation, (15) is the primary equation required to locate the horizon. Tod's proof of existence and uniqueness of solutions for this equation indicates that there must be a marginally trapped 2-surface on each slice $u=u_{0}$. However, in order for $\mathcal{H}^{-}$, the union of these surfaces, to be considered an apparent horizon, we require that $\mathcal{H}^{-}$be a non-timelike hypersurface, that is, that $N^{a} N_{a}$ is always non-negative. As shown by Collins [3] and Hayward [8], this is equivalent to the assumption that the directional derivative of the divergence of the ingoing null geodesics in the direction of future pointing outgoing null geodesics is negative. In fact, Hayward makes this assumption a part of the definition of an outer apparent horizon, and thus does not require a spacetime to be asymptotically flat in order for the outer apparent horizon to be selected. In the Robinson-Trautman spacetime, which is asymptotically flat, we prove that this assumption is not necessary; using the maximum principle and (16) it is straightforward to show that $N^{a} N_{a} \geq 0$ (details of the proof will be given elsewhere).

3.3. Properties of the horizon Equation (15) can be written in the form

$$
2 m e^{3 \Lambda}=e^{\Phi}\left(1-\Delta_{0} \Phi\right),
$$

where $e^{\Phi}=\mathfrak{R} e^{\Lambda}$. This gives a useful expression for the Gaussian curvature of the apparent horizon, $K_{T}=e^{-2 \Phi}\left(1-\Delta_{0} \Phi\right)$. It is then straightforward to show that the marginally trapped surfaces have the topology of $S^{2}$ :

$$
\chi_{T}=\frac{1}{2 \pi} \int_{S^{2}} K_{T} e^{2 \Phi} \omega_{0}=\frac{1}{2 \pi} \int_{S^{2}}\left[1-\Delta_{0} \Phi\right] \omega_{0}=2 .
$$

Furthermore, because $K_{T} e^{3 \Phi}=2 m e^{3 \Lambda}, K_{T}$ must be positive everywhere. Also, this relationship gives rise to some additional interesting integral quantities:

$$
\begin{aligned}
& \int_{S^{2}} m e^{2 \Lambda} \omega_{0}=4 \pi m \Leftrightarrow \int_{S^{2}} K_{T} e^{3 \Phi-\Lambda} \omega_{0}=8 \pi m, \\
& \int_{S^{2}} m e^{3 \Lambda-\Phi} \omega_{0}=2 \pi \quad \Leftrightarrow \quad \int_{S^{2}} K_{T} e^{2 \Phi} \omega_{0}=4 \pi \text {, } \\
& \int_{S^{2}} m e^{3 \wedge} \omega_{0} \geq 0 \quad \Leftrightarrow \quad \int_{S^{2}} K_{T} e^{3 \Phi} \omega_{0} \geq 0, \\
& \frac{d}{d u} \int_{S^{2}} m e^{3 \Lambda} \omega_{0} \leq 0 \quad \Leftrightarrow \quad \frac{d}{d u} \int_{S^{2}} K_{T} e^{3 \oplus} \omega_{0} \leq 0 .
\end{aligned}
$$

This is suggestive of the fact that the Robinson-Trautman equation can be rewritten entirely in terms of "horizon quantities". From (18), we have

$$
e^{2 \Lambda}=\left(\frac{1}{2 m} e^{\Phi}\left(1-\Delta_{0} \Phi\right)\right)^{2 / 3}
$$


and

$$
\Delta_{0} \Lambda=\frac{1}{3} \Delta_{0}\left(\Phi+\ln \left(1-\Delta_{0} \Phi\right)\right) .
$$

Using the Robinson-Trautman equation in the form

$$
\left(e^{2 \Lambda}\right)_{{ }_{u}}=-\frac{1}{12 m} \Delta_{0}\left(e^{-2 \Lambda}\left[1-\Delta_{0} \Lambda\right]\right),
$$

we can construct an equation in $\Phi$ alone. While rather messy, this formulation of the Robinson-Trautman equation is physically interesting as it describes in some sense the "evolution" of the apparent horizon of the white hole. The term "evolution" is used loosely here, since we expect the past apparent horizon to be non-timelike.

The properties of the area of the apparent horizon, $A_{\mathcal{I}}=\int_{S^{2}} e^{2 \Phi} \omega_{0}$, can also be derived from the horizon equations, Equations (15) to (17). Tod proved the isoperimetric inequality, $16 \pi M_{B}^{2} \geq A_{\mathcal{I}}$, for the area of the horizon [23]. In fact, $16 \pi M_{B}^{2} \geq A_{\mathcal{I}} \geq 16 \pi \mathrm{m}^{2}$, since, from the Hölder Inequality and (20),

$$
\begin{array}{rlrl}
\left(\int_{S^{2}} e^{2 \Phi} \omega_{0}\right)^{1 / 3}\left(\int_{S^{2}} e^{3 \Lambda-\Phi} \omega_{0}\right)^{2 / 3} & \geq \int_{S^{2}} e^{2 \Lambda} \omega_{0} \\
\Rightarrow & A_{\mathcal{I}}^{1 / 3}\left(\frac{2 \pi}{m}\right)^{2 / 3} & \geq 4 \pi \\
\Rightarrow & A_{\mathcal{I}} & \geq 16 \pi \mathrm{m}^{2}
\end{array}
$$

Since (16) implies that the apparent horizon must be a non-timelike hypersurface, that is, that $N^{a} N_{a} \geq 0$ always, we can show quite easily that the $A_{\mathcal{I}}$ must always decrease. From (15) and (17), we have

$$
\left(e^{2 \Phi}\right)_{u}+\Delta_{0}\left(e^{\Phi-\Lambda}\right)=-e^{\Phi+\Lambda}\left(N^{a} N_{a}\right)
$$

Thus

$$
\frac{d}{d u} A_{I}=\frac{d}{d u} \int_{S^{2}} e^{2 \Phi} \omega_{0}=-\int_{S^{2}} e^{\Phi+\Lambda}\left(N^{a} N_{a}\right) \omega_{0} \leq 0 .
$$

Thus we have shown that the past apparent horizon $\mathcal{H}^{-}$is a non-timelike hypersurface foliated by past marginally trapped surfaces $\mathcal{I}_{u}^{-}$whose surface area is a monotonically decreasing function of $u$.

\section{Numerical modelling}

We are currently undertaking to solve the apparent horizon equations in conjunction with the Robinson-Trautman equation numerically, and to use the numerical model 
to investigate further some of the properties of the apparent horizon. Our results at this stage demonstrate the nice properties of the horizon equations. We are initially solving the axisymmetric equations: thus the system is $1+1$ dimensional only. A full discussion of the numerical results will be presented elsewhere, but we give here a brief introduction to the work.

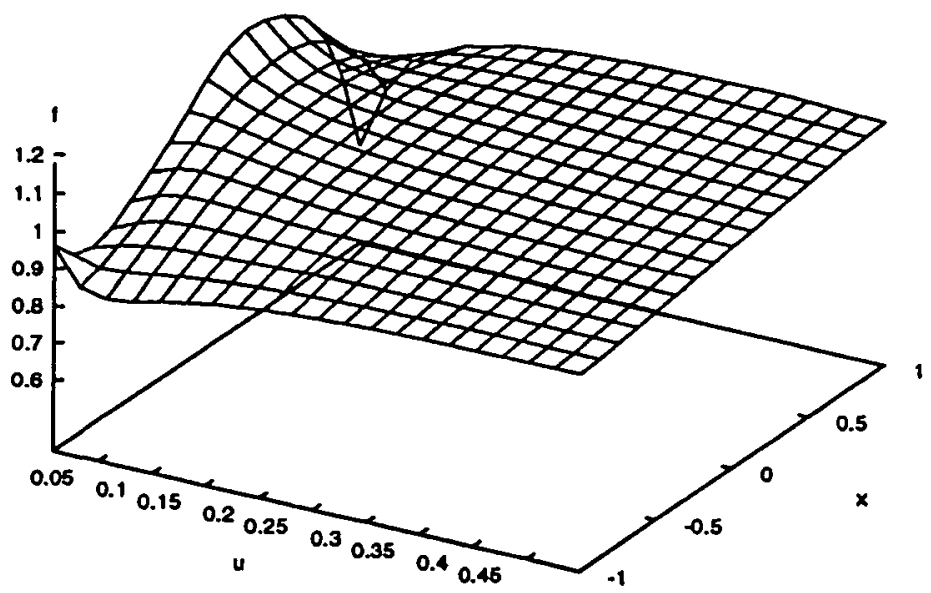

(a)

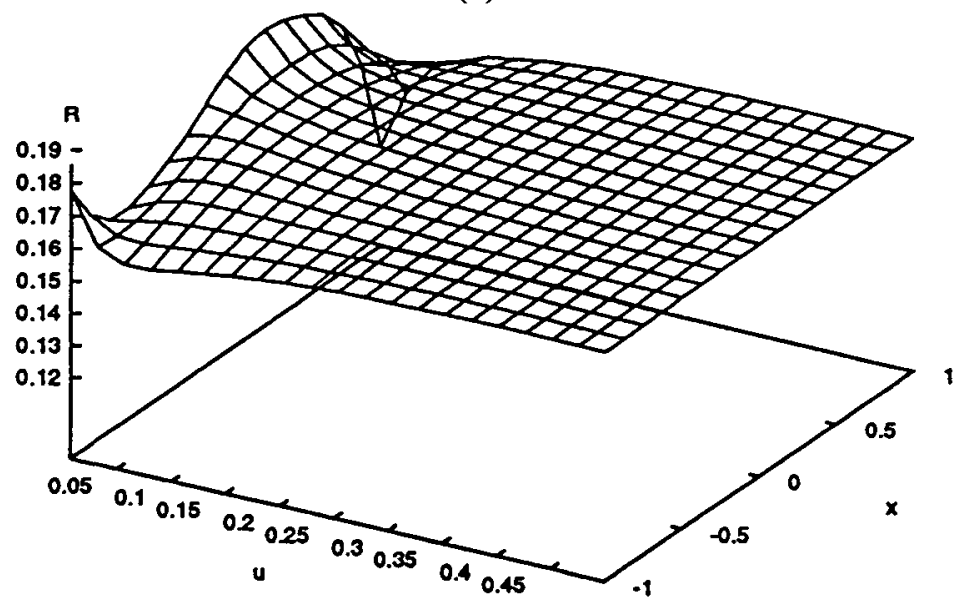

(b)

FIGURE 2. Numerical evolution of axisymmetric Robinson-Trautman spacetime and apparent horizon. (a) $f(x, y) \equiv P / P_{0}$, (b) $\Re(u, x)$

In order to find the apparent horizon, we first solve the Robinson-Trautman equation to find the "background spacetime". For this, we follow the work of Singleton $[20,22]$ and Prager and Lun [16], using exactly the Crank-Nicolson algorithm described by 
the latter. Next, on each 'time' slice $u=u_{0}$, we solve (15) using a Newton-Raphson iteration. That is, for the values of $P$ (or $\Lambda$ ) determined by the Robinson-Trautman equation, (15) is solved to find the function $\Re$ which describes the position of the marginally trapped surface. Because the convergence of the Newton-Raphson method is entirely dependent on making a good initial guess, we actually begin with the last timestep of the evolution, where the marginally trapped surface is known to be close to $r=2 m$, and solve for each time slice in turn, going backwards to $u=0$, taking as an initial guess for each slice the solution on the previous slice.

The example shown has the initial condition $f \equiv \frac{P}{P_{0}}=1 .-0.1 Y_{1,0}-0.2 Y_{2,0}-$ $0.3 Y_{3,0}$, where $Y_{t, 0}$ are spherical harmonics restricted to the axisymmetric case (that is, Legendre polynomials). For convenience we set $12 m=1$. Figure 2(a) shows a plot of $f$ against $x=\cos \theta$ evolving through $u$. It can be seen that the system settles down to equilibrium $\left(P=P_{0}\right)$ very quickly. Note that the equilibrium solution includes a component of the first harmonic in this case, so $f$ is of the form $f=a+b x$. The function $\Re$, representing the position of the marginally trapped surface $\mathcal{I}_{u}^{-}$, is plotted in Figure 2(b), also showing very smooth behaviour. $\Re$ is not subject to this ambiguity in the final state, and settles down as it should to $r=2 m$, which corresponds to the Schwarzschild equilibrium.

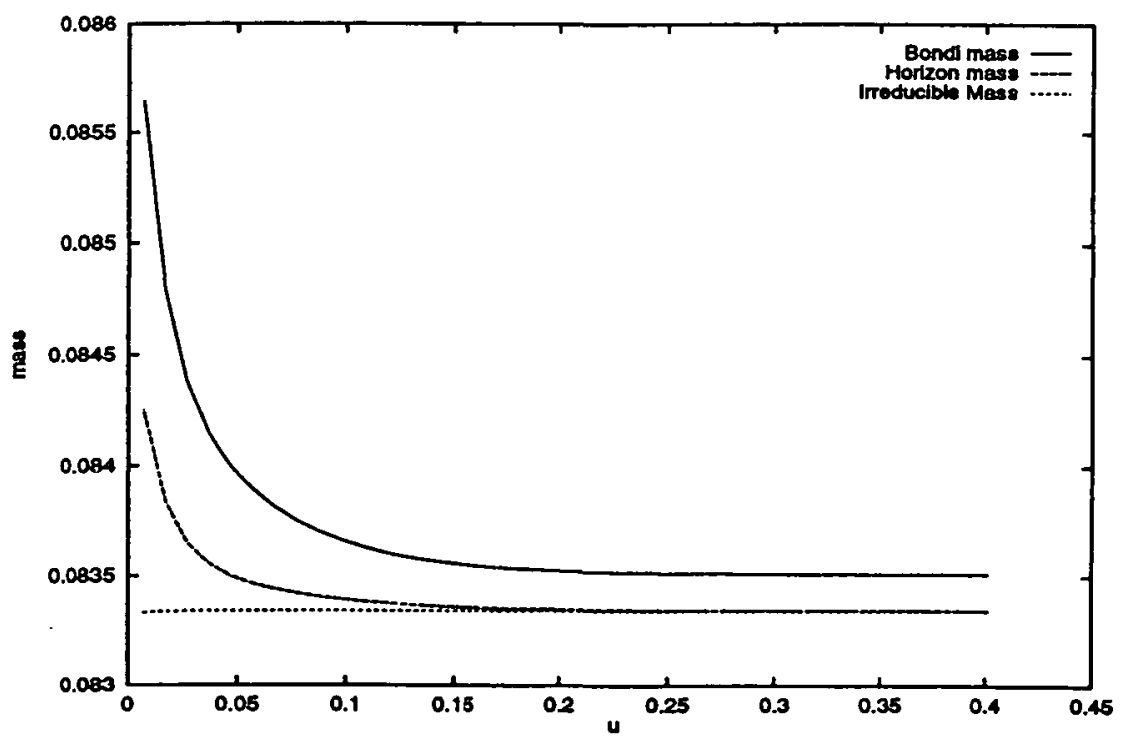

FIGURE 3. Monotonic decay of mass quantities in the numerical evolution of the Robinson-Trautman spacetime.

In Figure 3 the "horizon mass", defined as $M_{T}=\sqrt{A_{I} /(16 \pi)}$, is plotted as a function of $u$ with the Bondi mass and the irreducible mass, demonstrating the 
monotonicity of the horizon area, and the inequalities described above (21).

\section{Concluding remarks}

In this paper we have demonstrated the existence of the past apparent horizon in the vacuum asymptotically flat Robinson-Trautman spacetimes, and described some of its properties. Tod's proof of the existence of marginally past trapped surfaces [24], together with our result that they foliate a non-timelike hypersurface, allow us to call $\mathcal{H}^{-}$a past apparent horizon. It is pleasing to find that the Robinson-Trautman spacetimes, which are the simplest asymptotically flat radiating spacetimes, exhibit a well behaved apparent horizon structure. In more general radiating spacetimes, we would not expect the existence of apparent horizons to be guaranteed. This is demonstrated by examples such as the electrovac Robinson-Trautman [11] or the vacuum Bondi-Sachs spacetimes.

We have also shown that the surface area of the past apparent horizon decreases monotonically with the retarded time $u$. This result is a particular example of more general theorems about apparent horizon dynamics $[3,8]$. However, we have not required all the assumptions used in the proof of those theorems.

We have illustrated a method for describing apparent horizons analytically based on the construction of a suitable null tetrad for the spacetime - this method could be easily applied to other cases. In a future paper we will discuss further the numerical solution of the horizon equations, as means of investigating the physics of these spacetimes.

\section{Acknowledgements}

We would like to thank Dan Prager for helpful discussions and the Australian Research Council for financial support for this work.

This talk was given at the inaugural Australian General Relativity Workshop, A.N.U., Canberra, $26^{\text {th }}$ September 1994.

\section{References}

[1] P. Anninos, D. Bernstein, S. Brandt, J. Libson, J. Masso, E. Seidel, L. Smarr, W.-M. Suen and P. Walker, "Event horizons of numerical black holes", in General Relativity (MG7 Proceedings) (eds. R. Ruffini and M. Keiser), (World Scientific, Singapore, 1995).

[2] P. T. Chruściel, "Semi-global existence and convergence of solutions of the Robinson-Trautman (2-dimensional Calabi) equation", Commun. Math. Phys. 137 (1991) 289-313. 
[3] W. Collins, "Mechanics of apparent horizons", Phys. Rev. D 45 (1992) 495-498.

[4] G. Cook and J. M. York, "Apparent horizons for boasted and spinning black holes", Phys. Rev. D 41 (1990) 1077-1085.

[5] J. Foster and E. T. Newman, "Note on the Robinson-Trautman solutions", J. Math. Phys. 18 (1967) 189-194.

[6] G. Gibbons, S. Hawking, G. Horowitz and M. Perry, "Positive mass theorems for black holes", Commun. Math. Phys. 88 (1983) 295-308.

[7] S. W. Hawlang and G. F. R. Ellis, The Large Scale Structure of Space-time (Cambridge University Press, Cambridge, 1973).

[8] S. A. Hayward, “General laws of black-hole dynamics”, Phys. Rev. D 49 (1994) 6467-6474.

[9] C. Hoenselaers and Z. Perjés, "Remarks on the Robinson-Trautman solutions", Class. Quantum Grav. 10 (1993) 375-383.

[10] B. Lukács, Z. Perjés, J. Porter and I. Sebestyén, "Lyapunov functional approach to radiative metrics", Gen. Rel. Grav. 16 (1984) 691-701.

[11] A. W. Lun and E. W. M. Chow, "The role of the apparent horizon in the evolution of RobinsonTrautman Einstein-Maxwell space-times", in Confronting the Infinite (eds. A. Carey, W. Ellis, P. Pearce and A. Thomas), (World Scientific, Singapore, 1995).

[12] T. Nakamura, Y. Kojima and K. Oohara, "A method of determining apparent horizons in threedimensional numerical relativity", Phys. Lett. 106A (1984) 235-238.

[13] R. Penrose, "Naked singularities", Ann. NY Acad. Sci. 224 (1973) 115-134.

[14] R. Penrose and W. Rindler, Spinors and Space-time, Volume 1 (Cambridge University Press, Cambridge, 1984).

[15] Z. Perjés, "Robinson-Trautman space-times", in Proceedings of the 5th Marcel Grossman Meeting (eds. D. G. Blair and M. J. Buckingham), (World Scientific, Singapore, 1989).

[16] D. Prager and A. W. C. Lun, "Numerical integration of the Robinson-Trautman equation", in General Relativity (MG7 Proceedings) (eds. R. Ruffini and M. Keiser), (World Scientific, Singapore, 1995).

[17] A. D. Rendall, "Existence and asymptotic properties of global solutions of the Robinson-Trautman equation", Class. Quantum Grav. 5 (1988) 1339-1347.

[18] I. Robinson and A. Trautman, "Spherical gravitational waves", Phys. Rev. Lett. 4 (1960) 431-432.

[19] B. Schmidt, "Existence of solutions of the Robinson-Trautman equation and spatial infinity", Gen. Rel. Grav. 20 (1988) 65-70.

[20] D. Singleton, "Numerical evolution of the Robinson-Trautman equation", in Proceedings of the 5th Marcel Grossman Meeting (eds. D. G. Blair and M. J. Buckingham), (World Scientific, Singapore, 1989).

[21] D. Singleton, "On global existence and convergence of vacuum Robinson-Trautman solutions", Class. Quantum Grav. 7 (1990) 1333-1343.

[22] D. Singleton, "Robinson-Trautman solutions of Einstein's equations", Ph. D. Thesis, Monash University, 1990.

[23] K. P. Tod, “More on Penrose's quasi-local mass", Class. Quantum Grav. 3 (1986) 1169-1189.

[24] K. P. Tod, "Analogues of the past horizon in the Robinson-Trautman metrics", Class. Quantum Grav. 6 (1989) 1159-1163.

[25] M. A. J. Vandyck, "On the time evolution of some Robinson-Trautman solutions", Class. Quantum Grav. 4 (1987) 759-767. 